\title{
Agroforesterie et gestion durable des ressources naturelles pour l'atténuation et l'adaptation dans I'hinterland du Parc National de Kahuzi- Biega en RDC
}

\author{
Adolphine Muley-Byayuwa et Louis-Bernard Cheteu
}

\section{Contexte}

Les forêts en République Démocratique du Congo (RDC) couvrent une superficie estimée à 155,5 millions d'hectares et représentent près de la moitié des forêts tropicales humides d'Afrique (de Wasseige et al, 2009). Le taux annuel de déforestation en RDC est de $0,25 \%$ (FAO) et le taux de dégradation forestière entre 1990-2000 de 5,4\% (Chomitz et al.2006). La période de guerre et de troubles socio-politiques qu'a connue la RDC a occasionné une exploitation inappropriée des ressources naturelles et des menaces persistantes sur les espèces protégées. Avec un taux d'accroissement démographique les plus élevés de l'Afrique centrale 3,3\% (Defourny et al, 2011), la population humaine en RDC pourrait représenter une menace future sur les forêts, qui pourraient être soumises à une recrudescence de la déforestation et de la dégradation.

Conscient de ses responsabilités en tant que partie prenante à la Convention des Nations Unies sur les changements climatiques, le Gouvernement de la RDC s'est engagé à participer à l'effort commun visant le développement d'un cadre général d'intervention susceptible d'atténuer les effets résultant des changements climatiques observés tant au niveau local, régional que global.

Le projet intitulé 'Agroforesterie et gestions durables des ressources naturelles pour l'atténuation et l'adaptation dans l'hinterland du Parc National de Kahuzi-Biega (PNKB) en RDC'est initié par I'Union pour l'Emancipation des Femmes Autochtones Pygmées (UEFA). II fait partie d'une série de cinq projets pilotes conçus avec l'appui

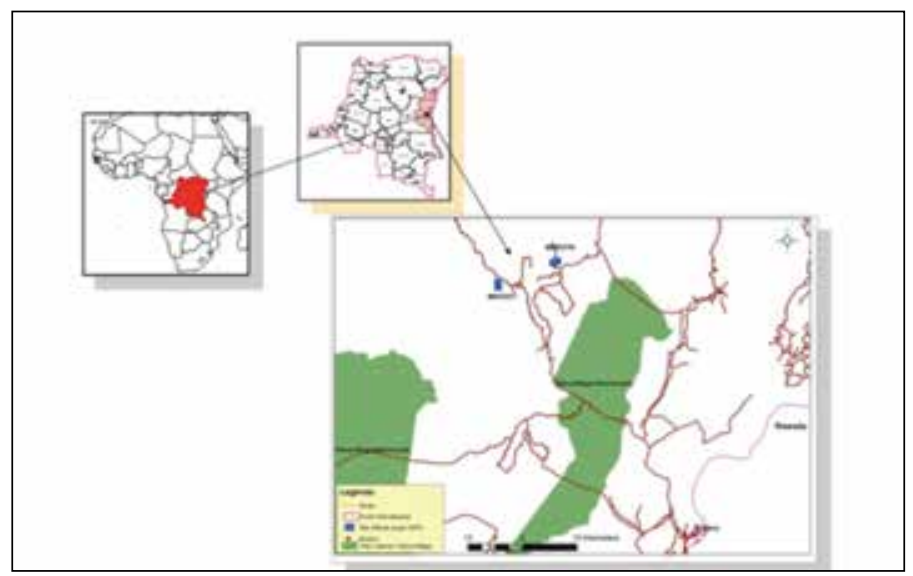

Figure 1. Carte de la zone du projet près du Parc National de Kahuzi-Biega. technique du CIFOR à travers le projet COBAM (Changement climatique et forêts dans le Bassin du Congo : synergie entre adaptation et atténuation). Le projet COBAM, ainsi que les projets pilotes, bénéficient de l'appui financier de la Banque africaine de développement (BAD) dans le cadre de l'aide accordée à la Communauté économique des États de l'Afrique centrale (CEEAC), pour financer le Programme d'appui à la conservation des écosystèmes du Bassin du Congo (PACEBCo).

\section{Le site}

La zone du projet est située à l'est de la RDC, province du sud Kivu à I'hinterland du Parc National de Kahuzi Biega dans le territoire de Kalehe à Bunyakiri, chefferie de Buhavu. Deux groupements ont été retenus par le projet à raison d'un village par groupement. II s'agit des villages Mafuo, avec une population estimée à 38933 habitants reparties en 6488 ménages (dans le groupement de Kalima) et Makuta avec une population estimée à 8500 habitants repartie en 1416 ménages (dans le groupement de Mubugu).

L'exploitation minière du coltan a provoqué une destruction du tissu social des habitants de la zone et une dévastation massive de l'environnement. La population se trouve dans un état désespéré, sans emploi, ni eau potable ou produits alimentaires, vivant dans l'insécurité. Les installations hospitalières, les écoles et routes existent mais sont dans un état de délabrement avancé et demandent une réhabilitation. La population de la zone d'étude pratique une économie de subsistance basée sur l'agriculture

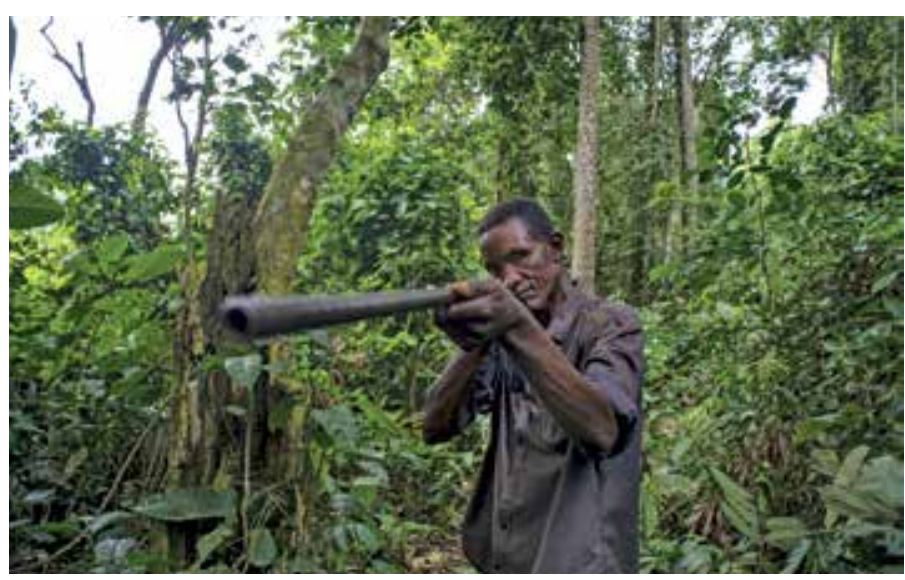

Figure 2. Un Chasseur. Photo Olivier Girard 


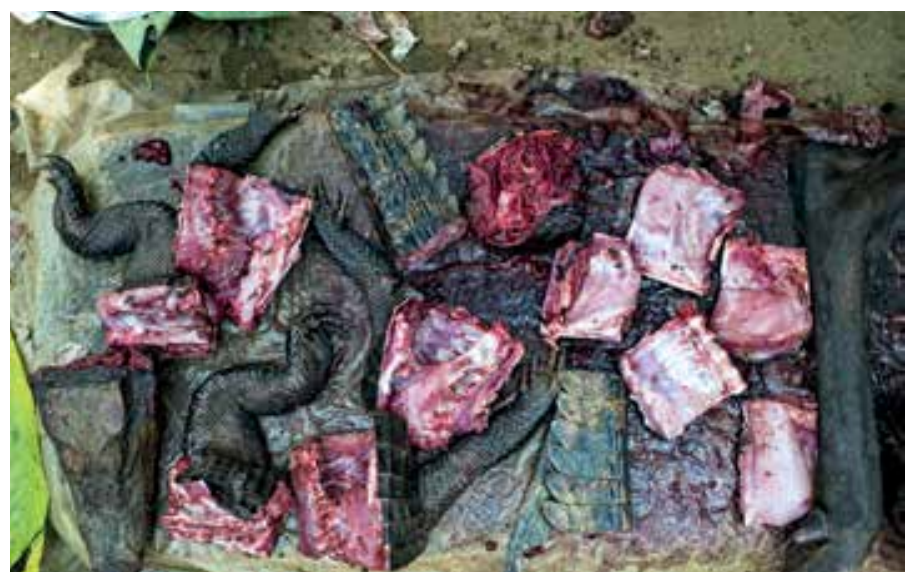

Figure 3. Viande de brousse. Photo Olivier Girard

itinérante et la chasse. Les principales sources de revenu pour les ménages comprennent l'agriculture, l'élevage, le petit commerce et l'artisanat. Le secteur de l'exploitation minière artisanale, en particulier l'exploitation des gisements de coltan, a le potentiel de donner un certain pouvoir économique à certains des groupes désavantagés et vulnérables et de contribuer ainsi aux efforts de réduction de la pauvreté dans la zone du projet, à condition que la sécurité et la paix reviennent (D'Souza, K., 2003). Les populations Pygmées vivent dans de petits villages situés près de la forêt et dont elles dépendent pour la chasse, la cueillette des fruits et la recherche de tubercules sauvages.

\section{Vulnérabilité des populations riveraines aux changements climatiques}

Les perturbations climatiques actuelles et passées ainsi que le pillage systématique des ressources ont contribué à la vulnérabilité des populations riveraines du PNKB. Celle-ci est aggravée par l'insécurité récurrente liée à la présence des groupes armés illégaux ainsi que des défis logistiques dus à la pénibilité de déplacements et à la quasi absence des moyens de communication (Ngeleza, R., 2012).

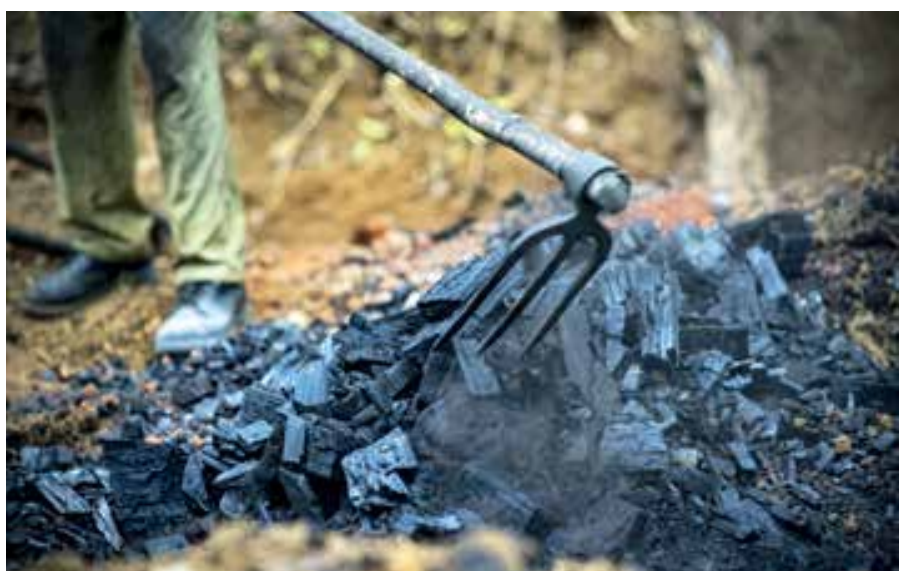

Figure 4. Elaboration artisanale du charbon.

Photo Olivier Girard

Les études de caractérisation de la vulnérabilité des populations locales menées dans le site par le CIFOR en 2012 (Bele et al. 2012) montrent que des perturbations climatiques fréquentes affectent négativement les rendements agricoles et le mode de vie des populations. Parmi ces perturbations liées au climat on a : le prolongement de la saison sèche, le prolongement de la saison des pluies, les vents violents, les poches sèches en saison de pluies et les pluies diluviennes.

Le tableau1 résume la perception des populations locales du niveau d'impact des évènements climatiques sur certaines activités de la zone du projet.

Comme on pourrait s'y attendre, l'agriculture vivrière serait donc très sensible aux aléas climatiques, plus particulièrement les plantes annuelles telles que le maïs, l'arachide ou le haricot. Certaines perturbations ont des effets plus prononcés que d'autres. C'est ainsi que les incertitudes en début de la saison pluvieuse ou l'apparition d'une poche de sècheresse à durée variable à la même période sont particulièrement néfastes sur le rendement agricole. La quasi disparition de la petite saison sèche serait une entrave à la préparation des champs pour la nouvelle saison agricole.

Tableau 1: Perception des populations de l'impact des perturbations climatiques sur les activités de production

\begin{tabular}{|c|c|c|c|c|c|c|}
\hline & Pluies diluviennes & $\begin{array}{l}\text { Incertitudes en } \\
\text { début } \\
\text { de la saison A }\end{array}$ & $\begin{array}{c}\text { Poche de } \\
\text { sècheresse après } \\
\text { le début } \\
\text { de la saison } \mathrm{A}\end{array}$ & $\begin{array}{c}\text { Raccourcissement } \\
\text { de la saison } \\
\text { pluvieuse A }\end{array}$ & $\begin{array}{l}\text { Vents violents } \\
\text { (ouragan) }\end{array}$ & $\begin{array}{l}\text { Disparition de la } \\
\text { petite saison sèche }\end{array}$ \\
\hline Manioc & -2 & 0 & 0 & 0 & -1 & -1 \\
\hline Arachide & -1 & -2 & -2 & -1 & 0 & -1 \\
\hline Maïs & 0 & -2 & -2 & -2 & -1 & -1 \\
\hline Haricot & -2 & -2 & -2 & 0 & -1 & -1 \\
\hline Concombres & 0 & -1 & -1 & 0 & 0 & -1 \\
\hline Banane & 0 & 0 & 0 & 0 & -2 & +2 \\
\hline Colocase & 0 & 0 & 0 & 0 & 0 & +2 \\
\hline Tilapia & -3 & 0 & 0 & 0 & 0 & 0 \\
\hline Poules & -3 & -1 & -1 & 0 & 0 & 0 \\
\hline Chèvres & -2 & -1 & -1 & -1 & 0 & 0 \\
\hline
\end{tabular}

Source : résultat des enquêtes menées dans le site du projet en juin 2012.

Légende : Intensité de l'impact de 0 à 3, avec 3 comme impact le plus élevé; qualité de limpact, positif(+) ou négatif(-). 


\section{Causes et agents de la déforestation et de la dégradation des forêts}

Une étude faite par le Ministère en charge de l'environnement et des forêts en RDC identifie et hiérarchise les causes de la déforestation et de la dégradation des forêts. Ainsi, comme causes directes, on cite l'agriculture itinérante sur brûlis, l'exploitation artisanale soit comme bois d'œuvre ou bois-énergie et l'exploitation minière artisanale. Les causes indirectes sont multiples, parmi lesquelles la croissance démographique, la pauvreté des masses paysannes et le déficit administratif (MECNT, 2012).

La zone de I'hinterland du PNKB présente des causes similaires, avec en plus la présence de quelques bandes armées résiduelles dans et autour du Parc qui s'attaquent aux ressources naturelles pour leur survie.

\section{Comment le projet apporte-t-il des solutions à ces problèmes ?}

Le projet vise un double objectif : réduire la vulnérabilité des populations et contribuer à réduire la concentration des gaz à effets de serre dans l'atmosphère (UEFA, 2011). Sa mise en œuvre va permettre de lutter contre la déforestation et la dégradation des forêts par la vulgarisation des pratiques agricoles et agro-forestières responsables, la promotion des foyers améliorés comme alternatives aux problèmes de bois énergie. Par ailleurs, le développement des activités susceptibles d'améliorer les conditions économiques et sociales de la population riveraine du parc national pourra contribuer à réduire la pauvreté dans la zone du projet.

Les activités identifiées de façon participative se regroupent en cinq composantes présentées dans le tableau 2 ci-après:

Tableau 2 : Résumé des composantes du projet et des bénéfices attendus

\begin{tabular}{|c|c|c|c|c|c|}
\hline \multirow{2}{*}{$\begin{array}{l}\text { Composantes } \\
\text { du projet }\end{array}$} & \multirow[b]{2}{*}{ Activités } & \multicolumn{3}{|c|}{ Profits } & \multirow[b]{2}{*}{$\begin{array}{l}\text { Bien-être des } \\
\text { communautés }\end{array}$} \\
\hline & & Atténuation & Adaptation & Biodiversité & \\
\hline $\begin{array}{l}\text { Amélioration des } \\
\text { pratiques agricoles et } \\
\text { agro-forestières; mise } \\
\text { en place de plantations } \\
\text { forestières. }\end{array}$ & $\begin{array}{l}\text { Ilnstallation de plantations } \\
\text { d'arbres fruitiers, } \\
\text { agroforestiers et forestiers } \\
\text { à partir des pépinières } \\
\text { communautaires. }\end{array}$ & $\begin{array}{l}\text { Augmentation du } \\
\text { stock de carbone } \\
\text { dans le paysage } \\
\text { considéré }\end{array}$ & $\begin{array}{l}\text { Protection contre } \\
\text { les effets du } \\
\text { changement } \\
\text { climatique (vent } \\
\text { violent, etc.) } \\
\text { Protection des sols, } \\
\text { disponibilité des } \\
\text { fruits en période de } \\
\text { récession. }\end{array}$ & $\begin{array}{l}\text { Amélioration de } \\
\text { la biodiversité } \\
\text { floristique; } \\
\text { Alternative au } \\
\text { braconnage }\end{array}$ & $\begin{array}{l}\text { Nouvelles sources } \\
\text { de revenus pour } \\
\text { les communautés à } \\
\text { travers les paiements } \\
\text { pour services } \\
\text { environnementaux }\end{array}$ \\
\hline $\begin{array}{l}\text { Promotion des foyers } \\
\text { améliorés }\end{array}$ & $\begin{array}{l}\text { Production et vulgarisation } \\
\text { des foyers améliorés }\end{array}$ & $\begin{array}{l}\text { Réduction de la } \\
\text { déforestation liée à } \\
\text { la recherche de bois } \\
\text { énergie }\end{array}$ & & & $\begin{array}{l}\text { Amélioration du } \\
\text { bien-être des } \\
\text { communautés à } \\
\text { travers la réduction } \\
\text { de la pénibilité des } \\
\text { activités domestiques }\end{array}$ \\
\hline $\begin{array}{l}\text { Promotion des activités } \\
\text { génératrices de revenus } \\
\text { et de réduction de la } \\
\text { pauvreté dans la zone } \\
\text { du projet }\end{array}$ & $\begin{array}{l}\text { Formation sur les } \\
\text { techniques d'élevage et } \\
\text { d'agriculture }\end{array}$ & $\begin{array}{l}\text { Conservation des } \\
\text { stocks de carbone } \\
\text { dans le paysage } \\
\text { considéré }\end{array}$ & $\begin{array}{l}\text { Contribution aux } \\
\text { actifs financiers, } \\
\text { sociaux et } \\
\text { socioéconomiques } \\
\text { renforçant la } \\
\text { capacité adaptative }\end{array}$ & $\begin{array}{l}\text { Alternatives à la } \\
\text { chasse }\end{array}$ & $\begin{array}{l}\text { Nouvelle source } \\
\text { de revenu pour les } \\
\text { communautés }\end{array}$ \\
\hline $\begin{array}{l}\text { Structuration des } \\
\text { communautés pour } \\
\text { la bonne gestion des } \\
\text { ressources naturelles }\end{array}$ & $\begin{array}{l}\text { Mise en place des comités } \\
\text { de gestion des ressources } \\
\text { naturelles }\end{array}$ & $\begin{array}{l}\text { Meilleure } \\
\text { gouvernance } \\
\text { pouvant assurer la } \\
\text { permanence des } \\
\text { forêts }\end{array}$ & $\begin{array}{l}\text { Amélioration de la } \\
\text { capacité adaptative } \\
\text { et du capital social }\end{array}$ & $\begin{array}{l}\text { Règles de } \\
\text { conservation et de } \\
\text { gestion durable } \\
\text { de la biodiversité } \\
\text { édictées et } \\
\text { respectées }\end{array}$ & $\begin{array}{l}\text { Maîtrise de la gestion } \\
\text { durable et meilleure } \\
\text { valorisation des } \\
\text { ressources naturelles }\end{array}$ \\
\hline $\begin{array}{l}\text { Plaidoyer auprès des } \\
\text { décideurs locaux, } \\
\text { provinciaux et } \\
\text { nationaux }\end{array}$ & $\begin{array}{l}\text { Présentation du cahier des } \\
\text { charges des communautés } \\
\text { locales quant au mode de } \\
\text { tenures et d'acquisition des } \\
\text { forêts des communautés } \\
\text { locales }\end{array}$ & $\begin{array}{l}\text { Meilleure prise en } \\
\text { compte des droits } \\
\text { des communautés } \\
\text { dans les activités } \\
\text { d'atténuation }\end{array}$ & $\begin{array}{l}\text { Amélioration du } \\
\text { capital social et } \\
\text { renforcement } \\
\text { de la capacité } \\
\text { d'adaptation. }\end{array}$ & $\begin{array}{l}\text { Négociation et } \\
\text { consensus sur les } \\
\text { principes et règles } \\
\text { de conservation et } \\
\text { gestion durable de } \\
\text { la biodiversité }\end{array}$ & $\begin{array}{l}\text { Renforcement } \\
\text { des droits des } \\
\text { communautés locales } \\
\text { sur la tenure et } \\
\text { l'acquisition des forêts } \\
\text { communautaires }\end{array}$ \\
\hline
\end{tabular}




\section{Les organisations de mise en œuvre du projet}

Le projet sera mis en œuvre par l'Union pour l'Emancipation de la Femme Autochtone, en abrégé «UEFA ».C'est une association créée en 1998 sans but lucratif, apolitique, non gouvernementale et non partisane de droit congolais, qui travaille pour la protection et la promotion des droits de l'Homme et des peuples autochtones en particulier, la protection de l'environnement, l'amélioration de I'habitat rural, l'agriculture - élevage, l'économie des ménages. Son siège se trouve à Bukavu en RDC.

Le CIFOR à travers le COBAM apporte un appui technique à la planification et la mise en œuvre des activités du projet et assure le suivi-évaluation de la mise en œuvre des activités du projet.

\section{Contacts}

Pour toute information complémentaire veuillez contacter :

- Adolphine MULEY BYAYUWA; Coordinatrice de UEFA; Bukavu, commune d'lbanda, avenue de l'Athénée, $n^{\circ} 3$;

Tél : +243994470 244/ +243998623642;

Courriel : uefafr@yahoo.fr

- Anne Marie Tiani ; Coordinatrice du projet COBAM: a.tiani@cgiar.org

- CIFOR Bureau régional pour l'Afrique Centrale : cifor.cameroon@cgiar.org

- PACEBCo : info@pacebco-ceeac.org

\section{Référence bibliographique}

Bele Y., Tiani A. M. et Pavageau C. 2012.Changement climatique et forêts du Bassin du Congo: synergies entre Adaptation et Atténuation (COBAM). Etude de caractérisation du site du paysage Kahuzi-Biega. Rapport interne. CIFOR, Yaoundé, Cameroun.

Chomitz, K.M., Buys P., de Luca, G., Thomas, T.S. et Wertz-Kanounnikoff, S. 2006. At logger heads? Agricultural expansion, poverty reduction, and environment in the tropical forests. Policy Research Report. Banque mondiale. Washington.

D'Souza, K. 2003. Etude d'échelle sur l'exploitation minière artisanale du coltan dans le parc national de Kahuzi-Biega, The Dian Fossey Gorilla Fund, Amstrong Wardell, Staffordshire, Royaume-Uni, 43p.

De Wasseige C., D. D., de Marcken P., Eba'a Atyi R., Nasi R. et Mayaux Ph. 2009. Etat des Forêts 2008. Les Forêts du Bassin du Congo. Luxembourg, Office des publications de l'Union européenne: $426 \mathrm{p}$.

Defourny, J.-P., Delhage, C., KibambeLubamba, J.P. 2011 Analyse quantitative des causes de la déforestation et de la dégradation des forêts en République démocratique du Congo. Rapport. FAO, Kinshasa, République Démocratique du Congo.

Ministère de l'Environnement, conservation de la nature et tourisme (MECNT) 2012. Etude qualitative sur les causes de la déforestation et de la dégradation des forêts en République Démocratique du Congo ; Kinshasa, République Démocratique du Congo. Version finale, août 2012.

Ngeleza, R. 2012. Le Paysage Maiko-Tayna-Kahuzi-Biega. Centre de recherche forestière internationale, Bogor, Indonésie.

UEFA 2011. Note d'idée de projet (NIP). Réponse à la vulnérabilité des écosystèmes adjacents du Parc National de Kahuzi-Biega (PNKB) en RD Congo. Union pour l'Emancipation de la Femme Autochtone. Bukavu, République Démocratique du Congo.

COBAM est mis en œuvre par le Centre pour la recherche forestière internationale (CIFOR), dans le cadre du support de la Banque africaine de développement $(B A D)$ à la Communauté économique des Etats de l'Afrique centrale (CEEAC), pour financer le Programme d'appui à la conservation des écosystèmes du Bassin du Congo (PACEBCo).
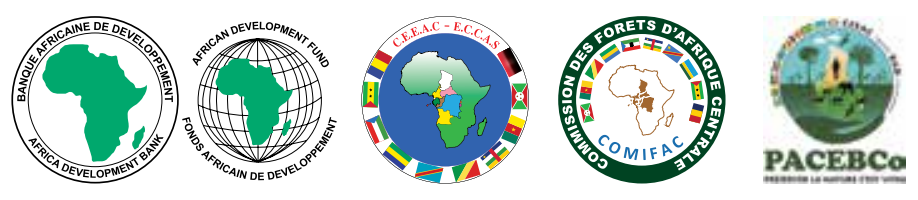

STOCKHOLM ENVIRONMENT INSTITUTE
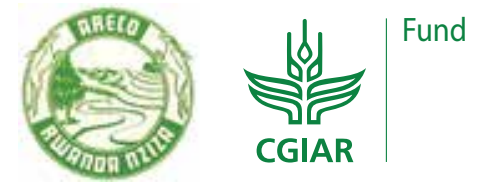

\section{cifor.org/cobam}

\section{Centre de recherche forestière internationale (CIFOR)}

Le CIFOR oeuvre en faveur du bien-être humain, de la conservation de l'environnement et de l'équité par sa recherche scientifique qui contribue à l'élaboration des politiques et des pratiques affectant les forêts dans les pays en développement. Le CIFOR est membre du Consortium du CGIAR. Son siège est situé à Bogor en Indonésie et il est également implanté en Asie, en Afrique et en Amérique latine. 